\title{
Inflamación y respuesta inmune innata: participación de las lipoproteínas de alta densidad
}

\author{
Damariz Marín-Palma' , Natalia Andrea Taborda², Silvio Urcuqui-Inchima ${ }^{3}$, Juan Carlos Hernandez ${ }^{4}$
}

\section{RESUMEN}

Las lipoproteínas de alta densidad (HDL) tienen como función transportar el exceso de colesterol desde los tejidos hacia el hígado, para ser excretado; $y$ de este modo contribuyen al control de las enfermedades cardiovasculares. Además, las HDL pueden modular la respuesta inmune, por sus propiedades anti-inflamatorias, antioxidantes y anti-apoptóticas, entre otras. A nivel celular, las HDL pueden modificar las balsas lipídicas, las cuales son determinantes en la activación de la respuesta inmune frente a patógenos o agentes extraños. En alounas patologías como la sepsis, las HDL participan mediando la eliminación del lipopolisacárido (LPS) a través de su captura y posterior eliminación en el hígado; esto conlleva a una modulación negativa de la expresión del TLR4, principal receptor del LPS. También se ha reportado que las HDL modulan la respuesta inflamatoria a través de la regulación de la activación de la cascada del complemento y la expresión de pentraxina 3. Finalmente, la función y los niveles de las HDL se han encontrado particularmente alterados en alogunas patologías infecciosas, ateroesclerosis $y$ sepsis, lo que se ha asociado con el progreso o la severidad de la enfermedad.

\section{PALABRAS CLAVE}

Colesterol; HDL; Inmunomodulación; Respuesta Inmune

\section{SUMMARY}

\section{Inflammation and innate immune response: role of high-density lipoprotein}

The main function of high-density lipoproteins (HDL) is to transport the excess of cholesterol from tissues to the liver, where it is excreted, thus decreasing the risk of atherosclerotic

1 Bióloga, Estudiante de Maestría, Infettare, Universidad Cooperativa de Colombia, Medellín, Colombia. Grupo Inmunovirología, Universidad de Antioquia, Medellín Colombia

2 Microbióloga y Bioanalista, MSc. Sci.Doc. Profesor investigador, Corporación Universitaria Remington, Medellín, Colombia. Grupo Inmunovirología, Universidad de Antioquia, Medellín Colombia

3 Biólogo. MSc. PhD. Profesor Asociado, Grupo Inmunovirología, Universidad de Antioquia, Medellín, Colombia

4 Microbiólogo y Bioanálista, Sci.Doc. Profesor investigador, Infettare, Universidad Cooperativa de Colombia, Medellín, Colombia. Grupo Inmunovirología, Universidad de Antioquia, Medellín Colombia

Correspondencia: Juan Carlos Hernández; juankhernandez@gmail.com

Recibido: octubre 3 de 2016

Aceptado: mayo 4 de 2017

Cómo citar: Marín-Palma D, Taborda NA, Urcuqui-Inchima S, Hernández JC. Inflamación y respuesta inmune innata: participación de las lipoproteínas de alta densidad. Iatreia. 2017 Oct-Dic;30(4):424-436. DOI 10.17533/udea.iatreia.v30n4a06 
plaques development and cardiovascular diseases establishment. Besides, HDL participates in different processes of the immune response, as its components have anti-inflammatory, anti-oxidative and antiapoptotic activities, among others. At cellular level, these lipoproteins have the ability to modify lipid rafts, critical micro-domains that participate in signaling pathways in response to pathogens. Likewise, it has been reported that HDL have a great impact in sepsis, as they eliminate the excess of LPS in blood and transport it to the liver for its elimination, and downregulate the expression of TLR4, main receptor of this molecule. Also, HDL can modulate humoral innate immune responses through regulating the activation of the complement pathway and the expression of pentraxin 3, resulting in the modulation of inflammatory processes. In fact, it has been reported that in several infectious diseases, atherosclerosis and sepsis, the level and function of HDL are altered, which associates with the progress of the diseases.

\section{KEY WORDS}

Cholesterol; HDL; Immunomodulation; Immune Response

\section{RESUMO}

Inflamação e resposta imune inata: participação das lipoproteínas de altadensidade

As lipoproteínas de alta densidade (HDL) têm a função de transportar o excesso de colesterol desde os tecidos até ofígado para aqui ser excretado e dessa forma contribuem no controlo das doenças cardiovasculares. Além disso, as HDL podemmodular a resposta imune devido as suas propriedades anti-inflamatórias, antioxidantes e antiapopticas, entre outras. Anívelcelular, as HDL podem modificar os rafts lipídicos, que são essências para a ativação da resposta imune contra patógenos ouagentes estranhos. Naloumas patologias como a septicemia, as HDL participam mediando a eliminação do lipopolissacarídeo (LPS) através da sua captura e posterior eliminação no fígado. Isto leva a uma modulação negativa da expressão de TLR4, orecetor principal do LPS. Também foi reportado que as HDL modulam a resposta inflamatória através da regulação da ativaçãoda cascata do sistema do complemento e a expressão de pentraxina 3. Por último, a função e os níveis das HDL têm sido especialmente encontrados alterados nalgumas doenças infeciosas, em aterosclerose e em septicemia, o qual esta associado aevolução ou a gravidade da doença.

\section{PALAVRAS CHAVE}

ColesteroI; HDL; Imunomodulação; Resposta Imune

\section{INTRODUCCIÓN}

Las lipoproteínas de alta densidad (HDL), comúnmente denominadas "colesterol bueno", son complejos macromoleculares heterogéneos encargados del transporte reverso de lípidos, desde los diferentes tejidos periféricos hacia el hígado, para remover el exceso de colesterol libre. Para llevar a cabo estas funciones, los diferentes tipos de lipoproteínas interactúan entre sí (1). Las HDL tienen además efectos pleiotrópicos en procesos como la vasodilatación (2) y la reducción de la oxidación de las lipoproteínas de baja densidad (LDL) (3), la inflamación, la apoptosis y la trombosis $(4,5)$. Igualmente, las HDL modulan los niveles de colesterol en células del sistema inmune como los macrófagos. Esto permite a su vez la regulación de la composición de los microdominios en la membrana plasmática $(6,7)$, denominados balsas Iipídicas, en los cuales se expresan receptores necesarios para la señalización celular, y por lo tanto, para la respuesta inmune (8). Además se ha descrito que durante el desarrollo de diversas patologías infecciosas, no solo hay un cambio en los niveles de las HDL, sino también en la composición y metabolismo de las lipoproteínas, lo que empeora su curso clínico (9). EI objetivo de este trabajo fue revisar los principales efectos pleiotrópicos reportados para las HDL en la respuesta inmune innata y su implicación en algunas patologías humanas.

\section{HDL y el transporte reverso}

EI principal componente estructural proteico de las HDL es la apoproteína A-I (ApoA-I), y en menor medida, ApoA-II, ApoA-IV, ApoC, ApoE, ApoJ y ApoM 
(Figura 1); adicionalmente posee enzimas como la lecitina colesterol acil-transferasa (LCAT), paraoxonasa-1 (PON-1), el factor activador de plaquetas acetilhidrolasa, y las proteínas de transferencia de ésteres de colesterol (CETP) y de fosfolípidos (PLTP) (10). También se ha descrito que diferentes miRNAs están asociados con las HDL (11) y que éstas pueden ser usadas como sistema de transporte para la entrega de miRNAs a células específicas (12). La fracción lipídica está compuesta por ésteres de colesterol, colesterol libre, triglicéridos y fosfolípidos. Las HDL pueden clasificarse según su tamaño, forma y contenido durante su biosíntesis, en partículas pequeñas discoidales (pre- $\beta 1$ У pre- $\beta 2$ ), esféricas intermedias (HDL3) y esféricas maduras (HDL2) (13). Éstas últimas interactúan con receptores scavenger en el hígado (SR-B1) para descargar el colesterol e iniciar su proceso de excreción (14).

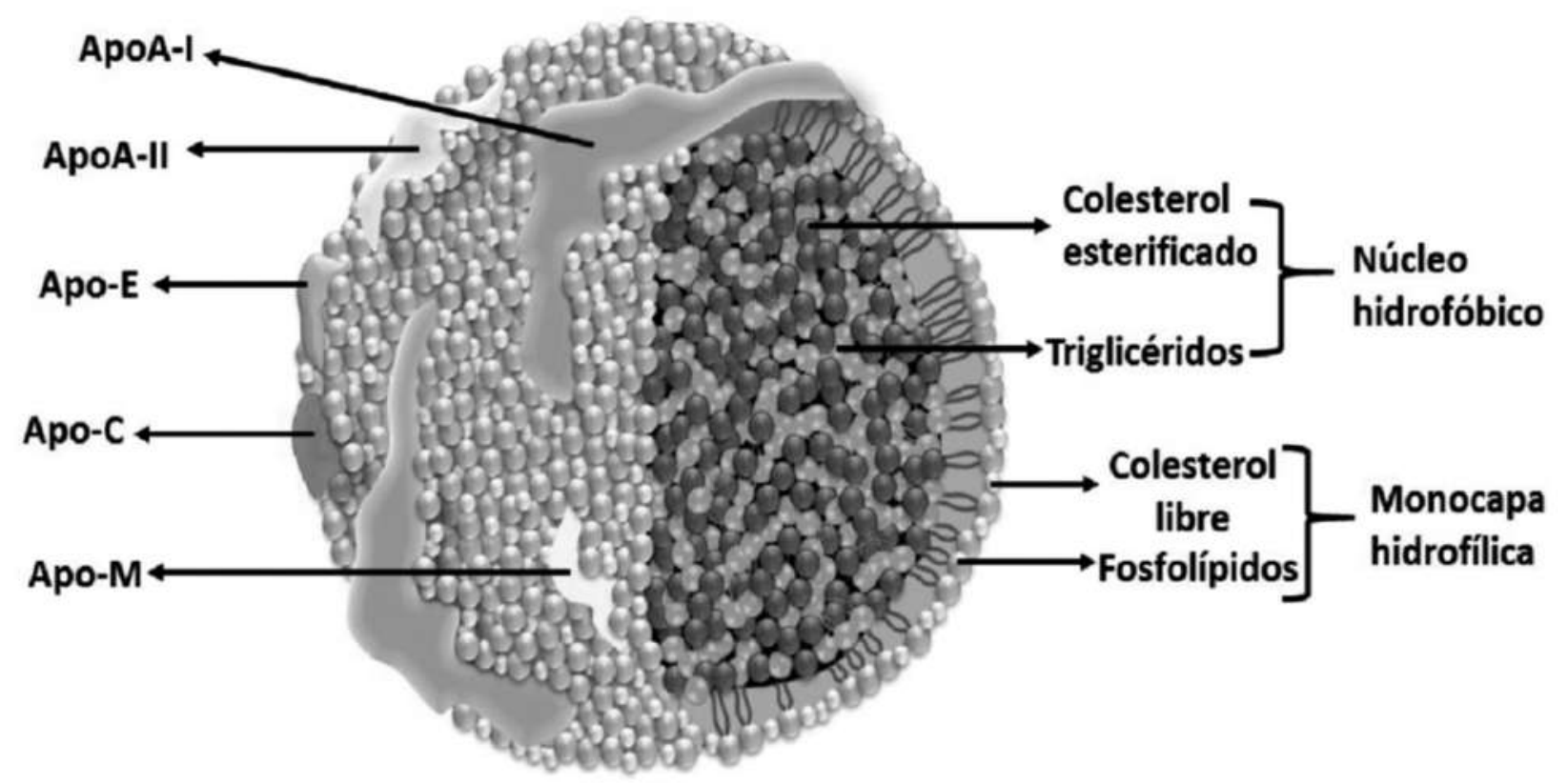

Figura 1. Estructura de las HDL maduras. Molécula esférica compuesta por una monocapa hidrofílica en la que se encuentran fosfolípidos, colesterol libre y apoproteínas, principalmente ApoA-I. Al interior, un núcleo hidrofóbico con triglicéridos y ésteres de colesterol.

Las HDL participan en un proceso denominado transporte reverso, el cual hace parte de la maduración de la macromolécula e implica la transferencia de apoproteínas y lípidos desde y hacia otras lipoproteínas (Tabla 1), y la toma de colesterol de membranas, revertiendo la acumulación en los tejidos. Para la formación de las HDL, Ia Apo-AI es secretada principalmente por el hígado y el intestino delgado en forma libre, (figura 2) y rápidamente obtiene pequeñas cantidades de fosfolípidos a través de la proteína transportadora dependiente de ATP 1 (ABCA1), incrementando el contenido lipídico y formando lo que se conoce como HDL pre- $\beta$ migrante o HDL naciente (15). En este proceso participan además diferentes enzimas como la LCAT, que cataliza la esterificación de los ácidos grasos mediante la transferencia de un grupo acil proveniente de la lecitina al colesterol libre, generando ésteres de colesterol hidrofóbicos, los cuales se desplazan hacia el interior de la lipoproteína $(16,17)$. Igualmente, Ia CEPT permite que ésteres de colesterol sean transferidos desde las HDL a lipoproteínas ricas en triglicériđos como LDL, y VLDL (very Iow density lipoproteins ). 
Tabla 1. Lipoproteínas que interactúan con las HDL (1)

\begin{tabular}{|c|c|c|c|}
\hline Lipoproteína & $\begin{array}{l}\text { Principales } \\
\text { apoproteínas }\end{array}$ & Función & Interacción con las HDL \\
\hline Quilomicrones & $\begin{array}{l}\text { ApoB-48, ApoA-I, } \\
\text { ApoA-II y ApoA-IV }\end{array}$ & $\begin{array}{l}\text { Transporte de los triacilgliceroles y del } \\
\text { colesterol obtenidos de la dieta, desde el } \\
\text { intestino. }\end{array}$ & $\begin{array}{l}\text { Transfieren entre sí apoproteínas y lípidos, } \\
\text { contribuyendo a la maduración de las HDL. }\end{array}$ \\
\hline VLDL & ApoB-100 & $\begin{array}{l}\text { Transporte a través de la sangre de los áci- } \\
\text { dos grasos y el colesterol (no esterificado) } \\
\text { producidos por el hígado. }\end{array}$ & $\begin{array}{l}\text { Adquieren ApoB y ApoE de las HDL cuando } \\
\text { está inmadura y a su vez transfieren fosfolí- } \\
\text { pidos y apoproteínas a las HDL. }\end{array}$ \\
\hline IDL & ApoB-100 & Da origen a la LDL. & Adquieren ApoE de las HDL. \\
\hline LDL & ApoB-100 & Transporte de lípidos a diferentes tejidos. & $\begin{array}{l}\text { Transfieren entre sí triacilgliceroles y coles- } \\
\text { terol esterificado. }\end{array}$ \\
\hline
\end{tabular}

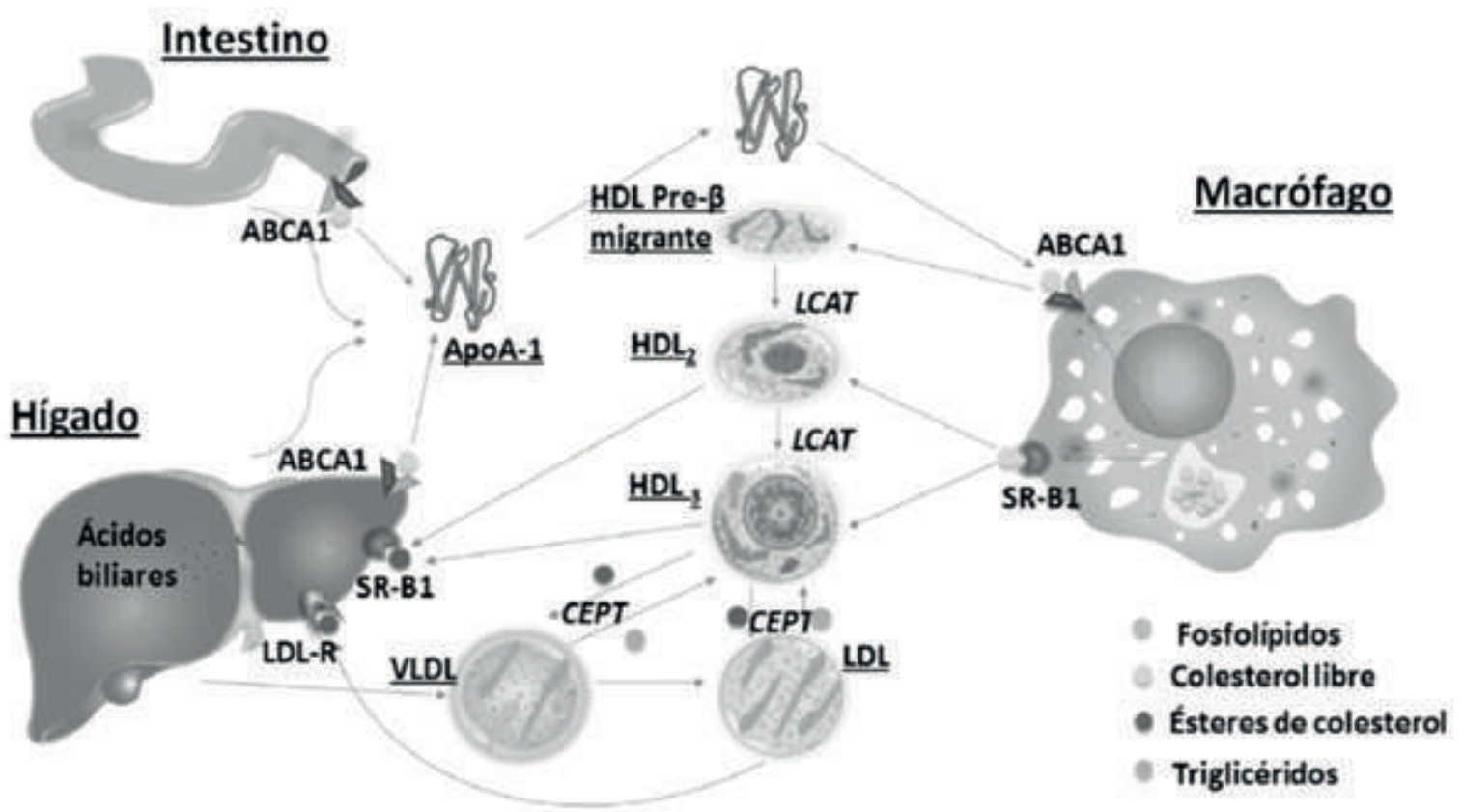

Figura 2. Metabolismo de las HDL. La ApoA-I es secretada principalmente por el hígado y el intestino y obtiene fosfolípidos, a través de la proteína ABCA1. ApoA-I viaja a tejidos extrahepáticos y obtiene colesterol libre, formando la pre-HDL. El colesterol es transformado a ésteres de colesterol, por acción de la LCAT, y migran al centro de la lipoproteína, dando origen a las formas de HDL esféricas maduras (HDL2 y HDL3). Estos complejos obtienen colesterol libre y fosfolípidos a través de ABCG1 y el receptor SR-B1. Además, intercambian ésteres de colesterol por triglicéridos y apoproteínas con las lipoproteínas LDL y VLDL (que contienen apoB-100) a través de la enzima (EPT, aumentando su contenido lipídico. El proceso es completado por dos vías: i) las HDL2 y HDL3 interactúan con el receptor SR-B1 presente en el hígado y transfiere los ésteres de colesterol o ii) a través de la transferencia de ésteres de colesterol de las HDL a las LDL y éstas a su vez se unen a los receptores de LDL en el hígado. Finalmente, los lípidos en el hígado son transformados en sales biliares que posteriormente son eliminados.

Las HDL maduras se unen al receptor SR-B1, que se encuentra principalmente en las balsas lipídicas y capta los ésteres de colesterol contenidos en las lipoproteínas, determinando los niveles de HDL en plasma y promoviendo el flujo de colesterol (14). Existen dos mecanismos por los cuales se da el transporte 
reverso de colesterol. El primero consiste en la unión de las HDL al receptor SR-B1, liberando el contenido de colesterol en el hígado (18); el segundo es a través de la transferencia de ésteres de colesterol a las VLDL y LDL (19), lo cual permite que las LDL se unan a su receptor específico y se dé la endocitosis de estas lipoproteínas.

\section{Papel de las HDL durante respuesta inflamatoria aguda}

Los niveles y la composición de las HDL se ven afectados por la acción de algunas citoquinas y moléculas liberadas durante los procesos inflamatorios. En este sentido, citoquinas pro-inflamatorias como TNF- $\alpha$ e IL-6 disminuyen la expresión de ApoA-I e inducen la expresión de la proteína amiloide A sérica (SAA), la cual cambia la composición de las HDL (20), afectando su capacidad antiinflamatoria y antioxidante (21). Se ha reportado que durante esta fase hay un incremento en la actividad de PLTP y una disminución en la actividad de LCAT, lo que lleva a una disminución de Ias HDL maduras (22). Otras alteraciones que se han evidenciado en la composición de las HDL durante la respuesta inflamatoria son el incremento de los niveles de triglicéridos, colesterol libre, ceramidas y glucosilceramidas, así como la disminución en los niveles de ésteres de colesterol y fosfolípidos, lo que en consecuencia modifica sus funciones (23).

Aunque durante la respuesta inflamatoria aguda se da un cambio en la composición de las HDL que disminuye su capacidad antiinflamatoria, se ha encontrado que algunos componentes inflamatorios pueden ser regulados por esta lipoproteína, como ocurre con los inflamasomas, que son complejos multiproteicos ensamblados en el citosol que favorecen la maduración de las citoquinas proinflamatorias de la familia de la IL-1 $\beta$ (24). Estos complejos pueden ser activados por cristales de colesterol, los cuales al ser fagocitados por los macrófagos, inducen una respuesta inflamatoria con la liberación de IL-1 $\beta$ e IL-18 $(25,26)$. Recientemente se ha demostrado que las HDL tienen la capacidad de disminuir la secreción de IL-1 en repuesta al estímulo con cristales de colesterol en macrófagos derivados de monocitos y la línea celular TPH-1; esta reducción podría asociarse con la capacidad de las HDL de modular negativamente la expresión transcripcional de NLRP3 e IL-1 $\beta$, así como de inducir una mayor estabilidad de la membrana lisosomal reduciendo la activación de la caspasa-1 y el daño mitocondrial (27). Por otra parte, dado el papel que tienen las HDL en el trasporte del colesterol, se han realizado diversos estudios para determinar su efecto en las lesiones ateroescleróticas. En ese sentido, ratones deficientes en ApoE, propensos a desarrollar procesos aterogénicos graves, en los que se silenció la expresión de NLRP3, presentan menor desarrollo de placas ateroescleróticas y mayor expresión transcripcional de los receptores $A B C A 1$ y $A B C G 1$, sugiriendo que hay un mayor flujo de colesterol en los macrófagos (28). En contraste, la administración de IL-18 en esos ratones, incrementa el tamaño de las lesiones ateroescleróticas (29), comprobando el vínculo que existe entre estas dos moléculas. Adicionalmente, la formación de cristales de colesterol en macrófagos alveolares es revertida por la adición de ApoA1 y ApoE (30), sugiriendo que las HDL podrían disminuir la activación del inflamasoma, y de esta manera, reducir la aparición de lesiones ateroscleróticas. Adicionalmente, nuestros estudios preliminares sugieren que las HDL promueven la degradación de los cristales de colesterol y contrarrestan su actividad pro-inflamatoria (Datos sin publicar).

Igualmente, se ha reportado que las HDL podrían modular el curso de algunas enfermedades con un componente inflamatorio, como el síndrome coronario agudo, en el cual se presentan bajos niveles de HDL, en comparación con los controles sanos (31). Dado que uno de los principales factores de riesgo para el desarrollo de enfermedades ateroscleróticas son los bajos niveles de HDL (32), y que estas enfermedades están asociadas con la activación de la respuesta inflamatoria, se sugiere que las HDL podrían participar en la protección de enfermedades cardiovasculares que están mediadas por la activación de moléculas pro-inflamatorias.

\section{Actividad antioxidante y anti-apoptótica de las HDL}

Las HDL juegan un papel importante en la inhibición de la oxidación de las LDL, ya que contienen apoproteínas y enzimas con actividad antioxidante, incluyendo PON-1, enzima que dirige la degradación de los ácidos grasos oxidados de las LDL, evitando que se desencadene la producción de citoquinas, mediadores inflamatorios y moléculas de adhesión celular, 
disminuyendo la respuesta inflamatoria (33). Los estudios en este campo han mostrado que existe una correlación negativa entre la expresión de PON-1 y la progresión de algunas patologías como la ateroesclerosis (34), enfermedades coronarias, diabetes mellitus y dislipidemias, en las cuales se presenta un incremento del estrés oxidativo (35). Así mismo, en modeIos murinos que no expresan PON-1, se ha observado una mayor progresión de las lesiones ateroescleróticas, así como menor actividad antioxidante de las HDL, en contraste con los ratones que sobre-expresan esta enzima (36), lo que refuerza el papel de esta enzima, uniđa a las HDL, en la reducción del estrés oxidativo. De igual forma, las HDL tienen la capacidad de inhibir la apoptosis en células endoteliales mediante la activación de la vía Akt/eNOS, las cuales son activadas por apoA-I y la esfingosina 1- fosfato (S1P-1, sphingosine 1- phosphate) al unirse a sus receptores SR-BI y S1P-2, respectivamente. Esta vía inhibe la inducción de apoptosis por la vía intrínseca, ayudando a mantener la integridad endotelial (5).

\section{Capacidad de unión y neutralización del LPS}

Una propiedad importante de las HDL, más específicamente de la ApoA-I, es la capacidad de unirse y neutralizar el lipopolisacárido (LPS) (37), que es reconocido por el TLR4 para activar la respuesta inflamatoria caracterizada por la producción de TNF- $\alpha$, IL- $1 \beta$ e IL- 6 (38). En modelos murinos transgénicos que expresan altos niveles de ApoA-I, se ha observado mayor sobrevida luego de que se induce endotoxemia por inyección peritoneal de LPS, respecto a los controles. Así mismo ratones deficientes en ApoA-I presentan mayor susceptibilidad al desarrollo de sepsis y una mayor producción de citoquinas proinflamatorias (39). De igual forma, ratones pre-tratados con HDL y retados con LPS, presentan mayor tasa de sobrevida y expresión reducida de marcadores de inflamación comparados con el control sin tratamiento, sugiriendo un papel protector de las HDL frente a la endotoxemia (40). Estos resultados concuerdan con los efectos observados en líneas celulares tratadas con HDL, en las cuales disminuye la secreción de citoquinas proinflamatorias en respuesta al LPS (Datos sin publicar).

Un factor importante en la respuesta inmune frente a las bacterias gram negativas es la proteína de unión al LPS (LBP), que media la activación del TLR4, aumentando la respuesta inflamatoria (41). Sin embaroo, se ha reportado que la proteína LBP puede tener un efecto dual en la respuesta al LPS (41): transfiriendo el LPS a las HDL, y de ahí a las LDL (42), o permitiendo su eliminación directa a través del hígado, disminuyendo la respuesta inflamatoria asociada al LPS (43). Los niveles plasmáticos de LPS son particularmente importantes en algunas enfermedades como la sepsis, en las cuales se ha observado que el receptor para las HDL, SR-B1, controla la respuesta frente a la endotoxemia, reduciendo la activación de NF-кB (44). De hecho, en ratones con deficiencia en el receptor SRB1, hay una menor sobrevida cuando se retan con LPS, sugiriendo un papel protector frente a la sepsis (45). Por otra parte, la expresión deI TLR4 se ve disminuida en monocitos activados por LPS y tratados con ApoA-I, en los cuales la integridad de las balsas lipídicas se ve afectada por la inducción de flujo de colesterol (46), lo que sugiere que las HDL pueden estar induciendo un cambio funcional en estas células, disminuyendo la respuesta inflamatoria frente al LPS. Sin embaroo, otros autores han reportado que el LPS puede contrarrestar los efectos de las HDL, reduciendo la expresión de ApoA-I y CETP, el transporte reverso y los efectos antiinflamatorios y antioxidantes de las HDL (47).

\section{Alteración de las balsas lipídicas por las HDL y sus implicaciones en la inmunidad innata}

Los macrófagos y las células dendríticas tienen un papel importante en la respuesta inmune. Para ello, en su membrana presentan balsas lipídicas que poseen microdominios ricos en colesterol, esfingolípidos $y$ proteínas, involucradas en procesos de señalización celular, transporte de proteínas $y$ adhesión celular (8). La función de las balsas lipídicas depende de su composición, por lo que su alteración lleva a la modulación de muchas vías de señalización de la respuesta inmune (48).

Se ha reportado que las HDL remueven el colesterol libre de las baIsas lipídicas, principalmente a través de las proteínas transportadoras ABCA1 y ABCG1 (49), proceso regulado por un heterodímero conformado por los receptores $\mathrm{X}$ de hígado y los receptores $\mathrm{X}$ retinoides. Este heterodímero induce la sobreexpresión de los genes que codifican para ApoA-I (50), y las proteínas transportadoras de colesterol en respuesta a oxiesteroles provenientes de la oxidación de las LDL, permitiendo una estimulación del transporte reverso del colesterol (51). Esta remoción de colesterol libre 
y de esfingomielina mediada por las HDL nacientes a través de ABCA1, lleva a un cambio de composición en las balsas lipídicas, lo que se ha asociado con una disminución de la expresión del marcador CD11b; induciendo alteraciones en la activación, la adhesión, y la dispersión y la miơración de neutrófilos y otras células $(48,49)$. Se ha encontrado que los macrófagos durante el desarrollo de procesos aterogénicos modulan el metabolismo de lípidos a través de la activación de mecanismos que remueven el exceso de colesterol, llevando a una regulación negativa de la expresión de mediadores inflamatorios como MCP-1 (monocyte chemoattractant protein 1) y CD11b (52). Adicionalmente, la alteración en la composición de las balsas lipídicas de los macrófagos lleva a una reducción de la expresión del complejo mayor de histocompatibilidad clase II, el cual es necesario para la activación de linfocitos T (53-55). De igual forma, las HDL aumentan la producción de prostaglandina E2 y de IL-10 por monocitos, lo que influye negativamente en su diferenciación hacia células dendríticas y de esta forma, se reduce la presentación antigénica (56).

Otros componentes importantes en la respuesta innata son los TLR, los cuales están involucrados en la formación de lesiones ateroescleróticas (57). Aunque estos receptores no se encuentran constitutivamente en las balsas lipídicas, una vez que son activados, se localizan en ellas para la señalización intracelular (58). Sin embargo, la alteración de la composición de colesterol de las baIsas lipídicas que ejerce la ApoA-I, lleva a una reducción de la expresión de alounos TLR, así como de los genes inducidos por estos (59). Otra vía por la cual se ha encontrado que las HDL puede modular la función de los TLR, es la activación del regulador transcripcional ATF3, el cual está involucrado en diferentes vías de señalización y se ha asociado con la inhibición de la expresión de genes blanco (60). Estos estudios sugieren que las HDL puede modular la función de los TLR mediante la alteración de los microdominios, evitando la migración de los TLR a las balsas lipídicas y posterior activación de las vías de señalización o modulando los reguladores negativos de su señalización, disminuyendo de esta forma el proceso inflamatorio.

\section{EI papel de las HDL en la función inmunológica de los esfingolípidos}

Uno de los principales componentes de las balsas lipídicas son los esfingolípidos, a partir de los cuales se generan ceramidas y esfingosina, la cual puede ser fosforilada por una kinasa de esfingosina (SphK, sphingosine kinasa) generando esfingosina 1 -fosfato (S1P) (61). Específicamente, se ha encontrado que S1P potencia el crecimiento y la sobrevida de diversos tipos celulares, modulando el ciclo celular $y$ el incremento de la síntesis de ADN (62). De iogual forma se asocia con la movilización de calcio intracelular independiente del inositol trifosfato (IP3) (63) y con la disminución de la permeabilidad vascular (64). De forma interesante, S1P podría intervenir en la modulación de las respuestas inflamatorias, tal como se evidenció en células $\mathrm{T}$ CD $4^{+}$de ratones deficientes en ShpK2, las cuales presentan un fenotipo hiperactivado, así como elevada capacidad proliferativa y de secreción de citoquinas en respuesta al estímulo con IL-2 (65). Además, durante la infección por el virus influenza, S1P reduce la producción de citoquinas pro-inflamatorias $y$ el reclutamiento de células innatas (66). En contraste, S1P puede también contribuir a respuestas inflamatorias y alérgicas, induciendo la degranulación de mastocitos (67). Además de inmunomodulador, S1P se ha encontrado correlacionado con los niveles de ApoA-I y apoAII (68) y es transportado por las HDL unido a ApoM (69). Aunque no se conoce el mecanismo por el cual las HDL remueven S1P de la membrana, se sabe que estas se unen, haciendo a S1P menos susceptible a la degradación y al unirse a su receptor, el receptor 3 de S1P, inhibe la miogración de macrófagos (70).

\section{Función de las HDL en la activación de la cascada del complemento}

El reconocimiento de patógenos por medio de receptores de la inmunidad innata lleva a la activación de las vías del complemento, que inducen la lisis bacteriana, opsonización, quimiotaxis y señalización a través de los TLR. Existe una correlación negativa entre los niveles de HDL y algunos componentes de la cascada del complemento, como C5b-C9 (71). De hecho, se ha descrito que las HDL inhiben la formación del complejo de ataque a membrana (72). Asimismo, estudios de proteómica han mostrado que en individuos con enfermedad arterial coronaria, las HDL se unen a las proteínas del complemento $\mathrm{C} 3 \mathrm{y}$ $\mathrm{C} 4$, en mayor medida que en los controles sanos (10). Cabe resaltar que el sistema del complemento ha sido asociado con aterogénesis, por lo que estos mecanismos 
pueden explicar dicha asociación (73). Este mecanismo tiene un papel importante en enfermedades como la ateroesclerosis, donde los cristales de colesterol activan la cascada del complemento, contribuyendo al proceso inflamatorio y de esta forma, a la progresión de la enfermedad. En efecto, a partir de muestras humanas, se observó que los niveles altos de HDL disminuyen la activación del complemento inducida por cristales de colesterol. Estos estudios sugieren que las HDL tiene un papel directo en la inhibición de la activación de la cascada del complemento, así como también un efecto indirecto, al disminuir el colesterol por transporte reverso y evitar la formación de cristales que pueden activar el complemento e inducir inflamación (74). En conclusión, estos hallazgos sugieren que las HDL y algunos componentes involucrados en su biosíntesis, puedan mediar los procesos de activación, diferenciación y función de las células de la inmunidad.

\section{participación de las HDL en enfermedades humanas}

Tanto las funciones metabólicas como inmunomoduladoras de las HDL pueden participar en la dinámica y progresión de las patologías humanas. Se han reportado niveles reducidos de HDL en enfermedades autoinmunes como lupus eritematoso sistémico (75), artritis reumatoide (76), enfermedad de Crohn y artritis psoriática (77), así como en otros tipos de patologías como ateroesclerosis (78) y sepsis (79). Las HDL con composición alterada, pueden desarrollarse en algunas patologías y contribuir con la severidad de la enfermedad. Tal es el caso de los pacientes con lupus eritematoso sistémico y artritis reumatoide (80), quienes presentan HDL con funciones proinflamatorias, las cuales tiene menor capacidad de transportar colesterol, así como alteración de su actividad antioxidante, debido a su menor contenido de ApoA-I y al aumento de enzimas como SAA (20). De igual forma, en pacientes con artritis reumatoide, existe una correlación negativa entre los anticuerpos generados contra las LDL oxidadas y los niveles de HDL, y una correlación positiva entre estos anticuerpos y el grado de inflamación (81). Cabe resaltar que en pacientes con lupus eritematoso, Ios anticuerpos contra Ias LDL oxidadas están asociados con un aumento en la formación de placas ateroescleróticas (82).

Otra enfermedad en la cual se ha reportado una alteración en las funciones anti-inflamatorias es la hiperhomocisteinemia. Los estudios señalan que el plasma de los pacientes con esta patología no tiene la capacidad de promover el flujo de colesterol desde los macrófagos cargados con lípidos y que la actividad de la PON1 (enzima contenida en las HDL) está disminuida, en comparación con controles sanos (83). De otro lado, en mujeres con síndrome antifosfolípido hay alteraciones en las funciones antioxidantes y antinflamatorias de las HDL, debido a que tienen menor capacidad de regular la producción de especies reactivas del oxígeno y de moléculas de adhesión como ICAM-1 y VCAM-1, así como baja capacidad de inducir la producción de óxido nítrico y menor actividad de la PON-1 (84).

En cuanto a enfermedades cardiovasculares, se han reportado dos polimorfismos en el gen que codifica para la PLTP, la cual que está involucrada en el proceso de maduración de las HDL, que se asocian con un aumento en el número de partículas de HDL de menor tamaño y con un menor riesgo de sufrir enfermedades cardiovasculares (85). En contraste, existen alounos desordenes genéticos que afectan directamente los niveles de HDL, tales como la enfermedad de Tangier, la cual es caracterizada por niveles muy bajos de HDL y el desarrollo de ateroesclerosis a temprana edad. Esta patología se debe a una deficiencia de la proteína $A B C A 1$ (86), la cual es crítica en la formación de las nuevas partículas de HDL. Finalmente, en la deficiencia de CETP, se presentan altos niveles de HDL, pero no se ha reportado su correlación en el control de la enfermedad (87).

Finalmente, tenemos las enfermedades infecciosas con un componente inflamatorio en su patogénesis, como las infecciones por los virus Dengue y VIH, entre otros. En ambos casos, las HDL se encuentran disminuidas, especialmente en aquellos pacientes con evidencias de progresión, es decir pacientes con signos de alarma en el caso del dengue, o pacientes con altas cargas virales $y$ bajos recuentos de linfocitos T CD4+, en el caso de VIH (datos sin publicar).

De esta forma, en las enfermedades donde se presenta alteración de las funciones antiinflamatorias de las HDL, se presenta exacerbación de la respuesta inflamatoria, lo que se relaciona directamente con una mayor severidad y progresión de estas enfermedades. 


\section{CONCLUSIÓN}

Estas evidencias sugieren que las HDL tiene un papel importante en la respuesta inmune innata y adaptativa, mediante la modulación de diferentes componentes del sistema inmune, lo que tiene un impacto importante en la respuesta frente a enfermedades como la ateroesclerosis y la endotoxemia. Este papel parece estar mediado por los componentes de las HDL, que pueden modular diferentes procesos como la migración de leucocitos, inflamación y presentación antigénica, además de la capacidad de transportar o eliminar mediadores como la S1P y el LPS, respectivamente. Por otra parte, la función de las HDL en el sistema inmune parece estar mediada por su función de promover el flujo de colesterol desde la célula, teniendo efecto en la expresión de algunos receptores importantes en la activación del sistema inmune adaptativo. En consecuencia, la alteración de estas funciones afecta el curso de diferentes enfermedades con un alto componente inflamatorio. Aunque aún se desconocen muchas vías y mecanismos que regulan la función de las HDL, su importancia en el control del desarrollo de diferentes enfermedades la señalan como un posible blanco terapéutico que debe continuar siendo objeto de investigación.

\section{AGRADECIMIENTOS}

Los autores agradecen al Dr. Walter Cardona por la lectura crítica del manuscrito y sus aportes para el mejoramiento del mismo. Colciencias 141565741029 , UdeA y uCC.

\section{CONFLICTOS DE INTERESES}

Los autores manifiestan no incurrir en ningún conflicto de intereses.

\section{REFERENCIAS BIBLIOGRÁFICAS}

1. Feingold KR, Grunfeld C. Introduction to Lipids and Lipoproteins. In: De Groot LJ, Chrousos G, Dungan K, Feingold KR, Grossman A, Hershman JM, et al., editors. Endotext [Internet]. South Dartmouth (MA): MDText.com, Inc.; 2000 [cited 2017 Mar]. Available from: http://www.ncbi.nIm.nih.gov/books/NBK305896/
2. Li XA, Titlow WB, Jackson BA, Giltiay N, NikolovaKarakashian M, Uittenbogaard A, et al. High density lipoprotein binding to scavenger receptor, Class B, type I activates endothelial nitric-oxide synthase in a ceramide-dependent manner. J Biol Chem. 2002 Mar;277(13):11058-63. DOI 10.1074/jbc.M1 10985200.

3. Uittenbogaard A, Shaul PW, Zuhanna IS, Blair A, Smart EJ. Hiog density lipoprotein prevents oxidized low density lipoprotein-induced inhibition of endothelial nitric-oxide synthase localization and activation in caveolae. J Biol Chem. 2000 Apr;275(15):11278-83.

4. Kameda T, Ohkawa R, Yano K, Usami Y, Miyazaki A, Matsuda K, et al. Effects of Myeloperoxidase-Induced Oxidation on Antiatherogenic Functions of HighDensity Lipoprotein. J Lipids. 2015;2015:592594. DOI 10.1155/2015/592594.

5. Nofer JR, Levkau B, Wolinska I, Junker R, Fobker M, von Eckardstein A, et al. Suppression of endothelial cell apoptosis by hiogh density lipoproteins (HDL) and HDL-associated lysosphingolipids. J Biol Chem. 2001 Sep;276(37):34480-5. DOI 10.1074/jbc.M103782200.

6. Zhu X, Owen JS, Wilson MD, Li H, Griffiths GL, Thomas MJ, et al. Macrophage ABCA1 reduces MyD88dependent Toll-like receptor trafficking to lipid rafts by reduction of lipid raft cholesterol. J Lipid Res. 2010 Nov;51(11):3196-206. DOI 10.1194/jlr.M006486.

7. Chowdhury SM, Zhu X, Aloor JJ, Azzam KM, Gabor $\mathrm{KA}, \mathrm{Ge} \mathrm{W}$, et al. Proteomic Analysis of ABCA1-Null Macrophages Reveals a Role for Stomatin-Like Protein-2 in Raft Composition and Toll-Like Receptor Signaling. Mol Cell Proteomics. 2015 JuI;14(7):185970. DOI 10.1074/mcp.M114.045179.

8. Simons K, Toomre D. Lipid rafts and siognal transduction. Nat Rer Mol Cell Biol. 2000 Oct;1(1):31-9. Erratum in: Nat Rev Mol Cell Biol 2001 Mar;2(3):216. DOI 10.1038/35036052.

9. Kapadia SB, Barth H, Baumert T, McKeating JA, Chisari $F V$. Initiation of hepatitis $C$ virus infection is dependent on cholesterol and cooperativity between CD81 and scavenger receptor B type I. J Virol. 2007 Jan;81(1):374-83. DOI 10.1128/JVI.01134-06.

10. Vaisar T, Pennathur S, Green PS, Gharib SA, Hoofnagle AN, Cheung MC, et al. Shotgun proteomics implicates protease inhibition and complement activation in the anti-inflammatory properties of HDL. J Clin Invest. 2007 Mar;117(3):746-56. DOI 10.1172/JCI26206.

11. Niculescu LS, Simionescu N, Sanda GM, Carnuta MG, Stancu CS, Popescu AC, et al. MiR-486 and miR-92a 
Identified in Circulating HDL Discriminate between Stable and Vulnerable Coronary Artery Disease Patients. PLoS One. 2015 Oct;10(10):e0140958. DOI 10.1371/journal.pone.0140958.

12. Vickers KC, Palmisano BT, Shoucri BM, Shamburek RD, Remaley AT. MicroRNAs are transported in plasma and delivered to recipient cells by high-density lipoproteins. Nat Cell Biol. 2011 Apr;13(4):423-33. DOI 10.1038/ncb2210. Erratum in: Nat Cell Biol. 2015 Jan; 17(1):104.

13. Gidez LI, Miller GJ, Burstein M, Slagle S, Eder HA. Separation and quantitation of subclasses of human plasma high density lipoproteins by a simple precipitation procedure. J Lipid Res. 1982 Nov;23(8):1206-23.

14. Gu X, Kozarsky K, Krieger M. Scavenger receptor class B, type I-mediated [3H]cholesterol efflux to high and low density lipoproteins is dependent on lipoprotein binding to the receptor. J Biol Chem. 2000 Sep;275(39):29993-30001.

15. Asztalos BF, de la Llera-Moya M, Dallal GE, Horvath KV, Schaefer EJ, Rothblat GH. Differential effects of HDL subpopulations on cellular ABCA1- and SRBI-mediated cholesterol efflux. J Lipid Res. 2005 Oct;46(10):2246-53. DOI 10.1194/jIr.M500187-JLR200.

16. Clay MA, Pyle DH, Rye KA, Barter PJ. Formation of spherical, reconstituted high density lipoproteins containing both apolipoproteins A-I and A-II is mediated by lecithin:cholesterol acyltransferase. J Biol Chem. 2000 Mar;275(12):9019-25.

17. Asztalos BF, Schaefer EJ, Horvath KV, Yamashita S, Miller M, Franceschini G, et al. Role of LCAT in HDL remodeling: investigation of LCAT deficiency states. J Lipid Res. 2007 Mar;48(3):592-9. DOI 10.1194/jlr. M600403-JLR200.

18. Acton S, Rigotti A, Landschulz KT, Xu S, Hobbs HH, Krieger M. Identification of scavenger receptor SR-BI as a high density lipoprotein receptor. Science. 1996 Jan;271(5248):518-20.

19. Hesler CB, Tall AR, Swenson TL, Weech PK, Marcel YL, Milne RW. Monoclonal antibodies to the Mr 74,000 cholesteryl ester transfer protein neutralize all of the cholesteryl ester and triglyceride transfer activities in human plasma. J Biol Chem. 1988 Apr;263(11):5020-3.

20. Tape C, Kisilevsky R. Apolipoprotein A-I and apolipoprotein SAA half-lives during acute inflammation and amyloidogenesis. Biochim Biophys Acta. 1990 Apr;1043(3):295-300.
21. Han CY, Tang C, Guevara ME, Wei H, Wietecha T, Shao B, et al. Serum amyloid A impairs the antiinflammatory properties of HDL. J Clin Invest. 2016 Jan;126(1):266-81. DOI 10.1172/JCI83475. Erratum in: J Clin Invest. 2016 Feb;126(2):796.

22. Pussinen PJ, Metso J, Malle E, Barlage S, Palosuo T, Sattler $\mathrm{W}$, et al. The role of plasma phospholipid transfer protein (PLTP) in HDL remodeling in acute-phase patients. Biochim Biophys Acta. 2001 Sep; 1533(2):153-63.

23. Sprandel MC, Hueb WA, Segre A, Ramires JA, KalilFilho R, Maranhão RC. Alterations in lipid transfers to HDL associated with the presence of coronary artery disease in patients with type 2 diabetes mellitus. Cardiovasc Diabetol. 2015 Aug; 14:107. DOI 10.1186/ s12933-015-0270-8.

24. Hernandez JC, Sirois CM, Latz E. Activation and regulation of the NLRP3 inflammasome. In: Couillin I, Pétrilli $\nabla$, Martinon F, editors. The Inflammasomes. Berlin: Springer Basel; 2011. p. 197-208. DOI 10.1007/978-3-0348-0148-5_13.

25. Duewell P, Kono H, Rayner KJ, Sirois CM, Vladimer G, Bauernfeind FG, et al. NLRP3 inflammasomes are required for atherogenesis and activated by cholesterol crystals. Nature. 2010 Apr;464(7293):1357-61. DOI 10.1038/nature08938. Erratum in: Nature. 2010 Jul;466(7306):652.

26. Rajamäki K, Lappalainen J, Oörni K, Välimäki E, Matikainen S, Kovanen PT, et al. Cholesterol crystals activate the NLRP3 inflammasome in human macrophages: a novel link between cholesterol metabolism and inflammation. PLoS One. 2010 Jul;5(7):e11765. DOI 10.1371/journal.pone.0011765.

27. Thacker SG, Zarzour A, Chen Y, Alcicek MS, Freeman LA, Sviridor DO, et al. High-density lipoprotein reduces inflammation from cholesterol crystals by inhibiting inflammasome activation. Immunology. 2016 Nov;149(3):306-19. DOI 10.1111/imm.12638.

28. Zheng F, Xing S, Gong Z, Mu W, Xing Q. Silence of NLRP3 suppresses atherosclerosis and stabilizes plaques in apolipoprotein E-deficient mice. Mediators Inflamm. 2014;2014:507208. DOI 10.1155/2014/507208.

29. Whitman SC, Ravisankar P, Daugherty A. Interleukin-18 enhances atherosclerosis in apolipoprotein E(-/-) mice through release of interferon-gamma. Circ Res. 2002 Feb;90(2):E34-8.

30. Kellner-Weibel G, Yancey PG, Jerome WG, Walser T, Mason RP, Phillips MC, et al. Crystallization of free 
cholesterol in model macrophage foam cells. Arterioscler Thromb Vasc Biol. 1999 Aug; 19(8):1891-8.

31. Wang L, Qu P, Zhao J, Chang Y. NLRP3 and downstream cytokine expression elevated in the monocytes of patients with coronary artery disease. Arch Med Sci. 2014 Aug;10(4):791-800. DOI 10.5114/ aoms.2014.44871.

32. Gordon T, Castelli WP, Hjortland MC, KanneI WB, Dawber TR. High density lipoprotein as a protective factor against coronary heart disease. The Framingham Study. Am J Med. 1977 May;62(5):707-14.

33. Harel M, Aharoni A, Gaidukov L, Brumshtein B, Khersonsky O, Meged R, et al. Structure and evolution of the serum paraoxonase family of detoxifying and anti-atherosclerotic enzymes. Nat Struct Mol Biol. 2004 May; 11(5):412-9. Erratum in: Nat Struct Mol Biol. 2004 Dec;11(12):1253. DOI 10.1038/nsmb767.

34. Bounafaa A, Berrougui H, Ghalim N, Nasser B, Bagri A, Moujahid A, et al. Association between Paraoxonase 1 (PON1) Polymorphisms and the Risk of Acute Coronary Syndrome in a North African Population. PLoS One. 2015 Aug; 10(8):e0133719. DOI 10.1371/ journal.pone.0133719.

35. Sanguinetti SM, Brites FD, Fasulo V, Verona J, Elbert A, Wikinski RL, Schreier LE. HDL oxidability and its protective effect against LDL oxidation in Type 2 diabetic patients. Diabetes Nutr Metab. 2001 Feb;14(1):27-36.

36. Rozenberg O, Rosenblat M, Coleman R, Shih DM, Aviram $M$. Paraoxonase (PON1) deficiency is associated with increased macrophage oxidative stress: studies in PON1-knockout mice. Free Radic Biol Med. 2003 Mar;34(6):774-84.

37. Henning MF, Herlax V, Bakás L. Contribution of the C-terminal end of apolipoprotein AI to neutralization of lipopolysaccharide endotoxic effect. Innate Immun. 2011 Feb;17(3):327-37. DOI 10.1177/1753425910370709.

38. Hoshino K, Takeuchi O, Kawai T, Sanjo H, Ogawa T, Takeda $\mathrm{Y}$, et al. Cutting edge: Toll-like receptor 4 (TLR4)-deficient mice are hyporesponsive to lipopoIysaccharide: evidence for TLR4 as the Lps gene product. J Immunol. 1999 Apr; 162(7):3749-52.

39. Guo L, Ai J, Zheng Z, Howatt DA, Daugherty A, Huang $B$, et al. High density lipoprotein protects against polymicrobe-induced sepsis in mice. J Biol Chem. 2013 Jun;288(25):17947-53. DOI 10.1074/jbc.M112.442699.

40. Levine DM, Parker TS, Donnelly TM, Walsh A, Rubin AL. In vivo protection against endotoxin by plasma high density lipoprotein. Proc Natl Acad Sci U S A. 1993 Dec;90(24):12040-4.

41. Geoner JA, Ulevitch RJ, Tobias PS. Lipopolysaccharide (LPS) signal transduction and clearance. Dual roles for LPS binding protein and membrane CD14. J Biol Chem. 1995 Mar;270(10):5320-5.

42. Levels JH, Marquart JA, Abraham PR, van den Ende AE, Molhuizen HO, van Deventer SJ, et al. Lipopolysaccharide is transferred from high-density to low-density lipoproteins by lipopolysaccharidebinding protein and phospholipid transfer protein. Infect Immun. 2005 Apr;73(4):2321-6. DOI 10.1128/ IAI.73.4.2321-2326.2005.

43. Casas AT, Hubsch AP, Rogers BC, Doran JE. Reconstituted high-density lipoprotein reduces LPS-stimulated TNF alpha. J Surg Res. 1995 Nov;59(5):544-52. DOI 10.1006/jsre.1995.1204

44. Guo L, Song Z, Li M, Wu Q, Wang D, Feng H, et al. Scavenger Receptor BI Protects against Septic Death through Its Role in Modulating Inflammatory Response. J Biol Chem. 2009 Jul;284(30):19826-34. DOI 10.1074/jbc.M109.020933.

45. Gilibert S, Galle-Treger L, Moreau M, Saint-Charles F, Costa S, Ballaire R, et al. Adrenocortical scavenger receptor class B type I deficiency exacerbates endotoxic shock and precipitates sepsis-induced mortality in mice. J Immunol. 2014 JuI;193(2):817-26. DOI 10.4049/jimmunol.1303164.

46. Smythies LE, White CR, Maheshwari A, Palounachari MN, Anantharamaiah GM, Chaddha M, et al. Apolipoprotein A-I mimetic 4F alters the function of human monocyte-derived macrophages. Am J Physiol Cell Physiol. 2010 Jun;298(6):C1538-48. DOI 10.1152/ ajpcell.00467.2009.

47. Khovidhunkit W, Kim MS, Memon RA, Shigenaga JK, Moser AH, Feingold KR, et al. Effects of infection and inflammation on lipid and lipoprotein metabolism: mechanisms and consequences to the host. J Lipid Res. 2004 Jul;45(7):1169-96.

48. Murphy AJ, Woollard KJ, Suhartoyo A, Stirzaker RA, Shaw J, Sviridor D, et al. Neutrophil activation is attenuated by high-density lipoprotein and apolipoprotein A-I in in vitro and in vivo models of inflammation. Arterioscler Thromb Vasc Biol. 2011 Jun;31(6):1333-41. DOI 10.1161/ATVBAHA.111.226258.

49. Murphy AJ, Woollard KJ, Hoang A, Mukhamedova N, Stirzaker RA, McCormick SP, et al. High-density 
lipoprotein reduces the human monocyte inflammatory response. Arterioscler Thromb Vasc Biol. 2008 Nov;28(11):2071-7. DOI 10.1161/ATVBAHA.108.168690.

50. Manna PR, Sennoune SR, Martinez-Zaguilan R, Slominski AT, Pruitt K. Regulation of retinoid mediated cholesterol efflux involves liver $\mathrm{X}$ receptor activation in mouse macrophages. Biochem Biophys Res Commun. 2015 Aug;464(1):312-7. DOI 10.1016/j. bbrc.2015.06.150.

51. Castrillo A, Joseph SB, Vaidya SA, Haberland M, Fogelman AM, Cheng G, et al. Crosstalk between LXR and toll-like receptor signaling mediates bacterial and viral antagonism of cholesterol metabolism. Mol Cell. 2003 Oct;12(4):805-16.

52. Moudry R, Spycher MO, Doran JE. Reconstituted high density lipoprotein modulates adherence of polymorphonuclear leukocytes to human endothelial cells. Shock. 1997 Mar;7(3):175-81.

53. Kabouridis PS, Janzen J, Magee AL, Ley SC. Cholesterol depletion disrupts lipid rafts and modulates the activity of multiple signaling pathways in $\mathrm{T}$ Iymphocytes. Eur J Immunol. 2000 Mar;30(3):95463. DOI 10.1002/1521-4141(200003)30:3<954::AIDIMMU1954>3.0.CO;2-Y.

54. Hiltbold EM, Poloso NJ, Roche PA. MHC class IIpeptide complexes and APC lipid rafts accumulate at the immunological synapse. J Immunol. 2003 Feb;170(3):1329-38.

55. Wang SH, Yuan SG, Peng DQ, Zhao SP. HDL and ApoA-I inhibit antigen presentation-mediated $\mathrm{T}$ cell activation by disrupting lipid rafts in antigen presenting cells. Atherosclerosis. 2012 Nov;225(1):105-14. DOI 10.1016/j.atherosclerosis.2012.07.029.

56. Kim KD, Lim HY, Lee HG, Yoon DY, Choe YK, Choi I, et al. Apolipoprotein A-I induces IL-10 and PGE2 production in human monocytes and inhibits dendritic cell differentiation and maturation. Biochem Biophys Res Commun. 2005 Dec;338(2):1126-36.

57. Xu XH, Shah PK, Faure E, Equils O, Thomas L, Fishbein MC, et al. Toll-like receptor-4 is expressed by macrophages in murine and human lipid-rich atherosclerotic plaques and upregulated by oxidized LDL. Circulation. 2001 Dec 18;104(25):3103-8.

58. Triantafilou M, Triantafilou K. Receptor cluster formation during activation by bacterial products. J Endotoxin Res. 2003;9(5):331-5. DOI 10.1179/096805103225002557.
59. White CR, Smythies LE, Crossman DK, Palgunachari MN, Anantharamaiah GM, Datta G. Regulation of pattern recognition receptors by the apolipoprotein A-I mimetic peptide 4F. Arterioscler Thromb Vasc Biol. 2012 Nov;32(11):2631-9. DOI 10.1161/ATVBAHA. 112.300167 .

60. De Nardo D, Labzin LI, Kono H, Seki R, Schmidt SV, Beyer $M$, et al. High-density lipoprotein mediates anti-inflammatory reprogramming of macrophages via the transcriptional regulator ATF3. Nat Immunol. 2014 Feb;15(2):152-60. DOI 10.1038/ni.2784.

61. Scanu AM, Edelstein C. HDL: bridging past and present with a look at the future. FASEB J. 2008 Dec;22(12):4044-54. DOI 10.1096/fj.08-117150.

62. Olivera A, Kohama T, Edsall L, Nava V, Cuvillier O, poulton $S$, et al. Sphingosine kinase expression increases intracellular sphingosine-1-phosphate and promotes cell growth and survival. J Cell Biol. 1999 Nov; 147(3):545-58.

63. Mattie M, Brooker G, Spiegel S. Sphingosine1-phosphate, a putative second messenger, mobilizes calcium from internal stores via an inositol trisphosphate-independent pathway. J Biol Chem. 1994 Feb;269(5):3181-8.

64. Li X, Stankovic M, Bonder CS, Hahn CN, Parsons M, Pitson SM, et al. Basal and angiopoietin-1-mediated endothelial permeability is regulated by sphingosine kinase-1. Blood. 2008 Apr;111(7):3489-97. DOI 10.1182/blood-2007-05-092148.

65. Samy ET, Meyer CA, Caplazi P, Langrish CL, Lora JM, Bluethmann H, et al. Cutting edge: Modulation of intestinal autoimmunity and IL-2 signaling by sphingosine kinase 2 independent of sphingosine 1-phosphate. J Immunol. 2007 Nov;179(9):5644-8.

66. Teijaro JR, WaIsh KB, Cahalan S, Fremgen DM, Roberts E, Scott F, et al. Endothelial cells are central orchestrators of cytokine amplification during influenza virus infection. Cell. 2011 Sep;146(6):980-91. DOI 10.1016/j. cell.2011.08.015.

67. Oskeritzian CA, Alvarez SE, Hait NC, price MM, Milstien S, Spiegel S. Distinct roles of sphingosine kinases 1 and 2 in human mast-cell functions. Blood. 2008 Apr;111(8):4193-200. DOI 10.1182/ blood-2007-09-115451.

68 Zhang B, Tomura H, Kuwabara A, Kimura T, Miura $\mathrm{S}$, Noda K, et al. Correlation of high density lipoprotein (HDL)-associated sphingosine 1-phosphate with serum levels of HDL-cholesterol and apolipoproteins. 
Atherosclerosis. 2005 Jan;178(1):199-205. DOI 10.1016/j.atherosclerosis.2004.08.024.

69. Liu M, Allegood J, Zhu X, Seo J, Gebre AK, Boudyguina $\mathrm{E}$, et al. Uncleared ApoM signal peptide is required for formation of large ApoM/sphingosine 1-phosphate (S1P)-enriched HDL particles. J Biol Chem. 2015 Mar;290(12):7861-70. DOI 10.1074/jbc.M114.631101.

70. Kimura T, Tomura H, Mogi C, Kuwabara A, Damirin A, Ishizuka T, et al. Role of scavenger receptor class $B$ type I and sphingosine 1-phosphate receptors in high density lipoprotein-induced inhibition of adhesion molecule expression in endothelial cells. J Biol Chem. 2006 Dec;281(49):37457-67. DOI 10.1074/jbc. M605823200.

71. Pasqui AL, Puccetti L, Bova G, Di Renzo M, Bruni F, Pastorelli $M$, et al. Relationship between serum complement and different lipid disorders. Clin Exp Med. 2002 May;2(1):33-8.

72. Jenne DE, Lowin B, Peitsch MC, Böttcher A, Schmitz G, Tschopp J. Clusterin (complement Iysis inhibitor) forms a high density lipoprotein complex with apolipoprotein A-I in human plasma. J Biol Chem. 1991 Jun;266(17):11030-6.

73. SpeidI WS, Kastl SP, Huber K, Wojta J. Complement in atherosclerosis: friend or foe? J Thromb Haemost. 2011 Mar;9(3):428-40. DOI 10.1111/j.15387836.2010.04172.X.

74. Niyonzima N, Samstad EO, Aune MH, Ryan L, Bakke SS, Rokstad AM, et al. Reconstituted High-Density Lipoprotein Attenuates Cholesterol Crystal-Induced Inflammatory Responses by Reducing Complement Activation. J Immunol. 2015 Jul;195(1):257-64. DOI 10.4049/jimmunol.1403044.

75. Formiga F, Meco JF, Pinto X, Jacob J, Moga I, Pujol R. Lipid and lipoprotein levels in premenopausal systemic lupus erythematosus patients. Lupus. 2001;10(5):359-63.

76. Yoo WH. Dyslipoproteinemia in patients with active rheumatoid arthritis: effects of disease activity, sex, and menopausal status on lipid profiles. J Rheumatol. 2004 Sep;31(9): 1746-53.

77. van Leuven SI, Hezemans R, Levels JH, Snoek S, Stokkers PC, Hovingh GK, et al. Enhanced atherogenesis and altered high density lipoprotein in patients with Crohn's disease. J Lipid Res. 2007 Dec;48(12):2640-6. DOI10.1194/jIr.M700176-JLR200.

78. Cui Y, Blumenthal RS, Flaws JA, Whiteman MK, Langenberg P, Bachorik PS, et al. Non-high-density lipoprotein cholesterol level as a predictor of cardiovascular disease mortality. Arch Intern Med. 2001 Jun; 161(11):1413-9.

79. Shor R, Wainstein J, Oz D, Boaz M, Matas Z, Fux A, et al. Low HDL levels and the risk of death, sepsis and malignancy. Clin Res Cardiol. 2008 Apr;97(4):227-33. DOI 10.1007/s00392-007-0611-z.

80. McMahon M, Grossman J, FitzGerald J, Dahlin-Lee E, Wallace DJ, Thong BY, et al. Proinflammatory highdensity lipoprotein as a biomarker for atherosclerosis in patients with systemic lupus erythematosus and rheumatoid arthritis. Arthritis Rheum. 2006 Aug;54(8):2541-9. DOI 10.1002/art.21976.

81. Peters MJ, van HaIm VP, Nurmohamed MT, Damoiseaux J, Tervaert JW, Twisk JW, et al. Relations between autoantibodies against oxidized low-density lipoprotein, inflammation, subclinical atherosclerosis, and cardiovascular disease in rheumatoid arthritis. J Rheumatol. 2008 Aug;35(8):1495-9.

82. McMahon M, Grossman J, Skaggs B, Fitzgerald J, Sahakian L, Ragavendra N, et al. Dysfunctional proinflammatory high-density lipoproteins confer increased risk of atherosclerosis in women with systemic Iupus erythematosus. Arthritis Rheum. 2009 Aug;60(8):2428-37. DOI 10.1002/art.24677.

83. Holven KB, Aukrust P, Retterstøl K, Otterdal K, Bjerkeli $\nabla$, Ose L, et al. The antiatherogenic function of HDL is impaired in hyperhomocysteinemic subjects. J Nutr. 2008 Nov;138(11):2070-5. DOI 10.3945/jn.108.090704.

84. Charakida M, Besler C, Batuca JR, Sangle S, Marques $\mathrm{S}$, Sousa M, et al. Vascular abnormalities, paraoxonase activity, and dysfunctional HDL in primary antiphospholipid syndrome. JAMA. 2009 Sep;302(11):1210-7. DOI 10.1001/jama.2009.1346.

85. Vergeer M, Boekholdt SM, Sandhu MS, Ricketts SL, Wareham NJ, Brown MJ, et al. Genetic variation at the phospholipid transfer protein locus affects its activity and high-density lipoprotein size and is a novel marker of cardiovascular disease susceptibility. Circulation. 2010 Aug;122(5):470-7. DOI 10.1161/CIRCULATIONAHA.109.912519.

86. Brunham LR, Kang MH, Van Karnebeek C, Sadananda SN, Collins JA, Zhang LH, et al. Clinical, Biochemical, and Molecular Characterization of Novel Mutations in ABCA1 in Families with Tangier Disease. JIMD Rep. 2015;18:51-62. DOI 10.1007/8904_2014_348.

87. Mabuchi H, Nohara A, Inazu A. Cholesteryl ester transfer protein (CETP) deficiency and CETP inhibitors. Mol Cells. 2014 Nov;37(11):777-84. DOI 10.14348/ molcells.2014.0265. 